\title{
Towards the solution of the relativistic gravitational radiation reaction problem for binary black holes
}

\author{
Carlos O Lousto \\ Albert-Einstein-Institut, Max-Planck-Institut für Gravitationsphysik, Am Mühlenberg 1, \\ D-14476 Golm, Germany \\ and \\ Instituto de Astronomía y Física del Espacio, CONICET, Buenos Aires, Argentina
}

Received 5 July 2001

Published 17 September 2001

Online at stacks.iop.org/CQG/18/3989

\begin{abstract}
Here we present the results of applying the generalized Riemann $\zeta$-function regularization method to the gravitational radiation reaction problem. We analyse in detail the head-on collision of two non-spinning black holes with an extreme mass ratio. The resulting reaction force on the smaller hole is repulsive. We discuss the possible extensions of these method to generic orbits and spinning black holes. The determination of corrected trajectories allows us to add second perturbative corrections with the consequent increase in the accuracy of computed waveforms.
\end{abstract}

PACS numbers: 0430, 0425N, 0425, 0470B

There has been increasing astronomical evidence over the previous 30 years in favour of the hypothesis that supermassive black holes, lying in the centre of galaxies are a common phenomenon in our Universe. They are thought to be the main engines of quasars, $\mathrm{x}$-ray sources in the core of galaxy superclusters and gamma-ray bursters. There is even compelling evidence that our own galaxy shelters a black hole of $3 \times 10^{6}$ solar masses in its centre [1]. Although compelling, this is still indirect evidence of the existence of black holes. It appears that their characteristic gravitational wave signature provides the best way to determine their definitive existence. The LISA project [2] offers an excellent opportunity to match theory and observation in order to reach such a goal. From the characteristics of this space detector, the main astrophysical sources expected to be 'seen' are precisely supermassive black holes accreting stars close to the galactic centre. This scenario offers the possibility to use perturbation theory around a single black hole as the appropriate theoretical model to compute gravitational radiation from such extreme mass ratio binary systems.

Perturbation theory had already reached maturity in the early 1970s [3,4]. Linearized Einstein equations have been successfully combined into wave equations for the two degrees of freedom of the gravitational field propagating in the curved background of a massive black 
hole. The source terms depend on the trajectory of a test particle moving along a geodesic of the background metric. There remained one important problem to be solved: the changes induced in the trajectory of the particle due to the radiation generated by the orbital motion of the same particle around the massive hole. Progress along this line always stumbled with the infinities appearing in the equation of motion of the particle when perturbations are evaluated, precisely at the location of the particle. A consistent regularization method was lacking until recently, when Mino et al [5] provided a renormalized equation of motion using two different methods: (a) the Hadamard regularization and (b) an asymptotic matching of the near- and far-metric expansions. Quinn and Wald [6] assume an axiomatic approach to obtain the same equation of motion independently. While the above work provides well founded formal expressions, they appear impractical as yet when dealing with concrete computations since they rely on the use of the harmonic gauge. Here we present an alternative, practical regularization method based on the analytically continued Riemann $\zeta$-function.

A test particle with rest mass $m_{0}$ is represented here by the energy-momentum tensor

$$
T^{\mu \nu}=m_{0} \frac{U^{\mu} U^{\nu}}{U^{0} r^{2}} \delta\left[r-r_{p}(t)\right] \delta^{(2)}\left[\Omega_{p}\right] .
$$

Since the only 'forces' acting on the particle are gravitational, the equation of motion of a test particle is given by the geodesic equation

$$
\frac{\mathrm{d}^{2} x_{p}^{\mu}}{\mathrm{d}^{2} \lambda}+\Gamma_{\alpha \beta}^{\mu}\left(x_{p}\right) \frac{\mathrm{d} x_{p}^{\alpha}}{\mathrm{d} \lambda} \frac{\mathrm{d} x_{p}^{\beta}}{\mathrm{d} \lambda}=0 .
$$

It is precisely when one wants to evaluate the Christoffel symbols at the location of the particle that divergences appear. They originate in the Dirac delta function representation we give to the source term (1) of Einstein's equations.

In [7-9] we studied the head-on collision of two Schwarzschild (non-spinning) black holes with masses $M$ and $m$, respectively. We treat this problem as perturbations of the Schwarzschild background. The only expansion parameter being $m / M$, assumed to be much less than one. The symmetry of the background allows us to decompose metric perturbations into spherical harmonics $Y_{\ell m}(\theta, \phi)$ and the gravitational degrees of freedom satisfy the wave equation

$$
-\frac{\partial^{2} \psi_{\ell}}{\partial t^{2}}+\frac{\partial^{2} \psi_{\ell}}{\partial r^{* 2}}-V_{\ell}(r) \psi_{\ell}=\mathcal{S}_{\ell}(r, t)
$$

where $r^{*} \equiv r+2 M \ln (r / 2 M-1), V_{\ell}$ is the Zerilli potential and $\mathcal{S}_{\ell}(r, t)$, is the source term generated by the small hole $[7,8]$.

From the waveform $\psi_{\ell}(r, t)$ one can reconstruct all perturbed metric coefficients in the Regge-Wheeler gauge. One can then prove that all metric coefficients are $C^{0}$ at $r_{p}$ for every $\ell$. The divergences reappear when trying to add up all multipole contributions [10]. To see this more explicitly, let us consider the $r$ and $t$ components (the only non-trivial for the head-on case) of the geodesic equation (2) and combine them into a single equation for $r(t)$

$$
\frac{\mathrm{d}^{2} r}{\mathrm{~d} t^{2}}=\Gamma_{r r}^{t}\left(\frac{\mathrm{d} r}{\mathrm{~d} t}\right)^{3}+\left(2 \Gamma_{t r}^{t}-\Gamma_{r r}^{r}\right)\left(\frac{\mathrm{d} r}{\mathrm{~d} t}\right)^{2}+\left(\Gamma_{t t}^{t}-2 \Gamma_{t r}^{r}\right)\left(\frac{\mathrm{d} r}{\mathrm{~d} t}\right)-\Gamma_{t t}^{r}
$$

Linearization of this equation and subtraction of the zeroth-order geodesic gives the deviation of the trajectory $\Delta r_{p}(t)$ from the zeroth-order one,

$$
\dot{r}_{p}(t)=\partial_{t} z_{p}=-\left(1-2 M / z_{p}\right) \sqrt{\frac{2 M / z_{p}-2 M / z_{0}}{1-2 M / z_{0}}},
$$


directly in terms of Schwarzschild coordinates, ready for further applications

$$
\Delta \ddot{r}_{p}=A \Delta r_{p}+B \Delta \dot{r}_{p}+C
$$

where

$$
\begin{aligned}
A & =\frac{2 M}{r_{p}^{3}}\left[3-\frac{3 M}{r_{p}}-\frac{\left(1-3 M / r_{p}\right) \dot{r}_{p}^{2}}{\left(1-2 M / r_{p}\right)^{2}}\right] \\
B & =\frac{6 M \dot{r}_{p}}{r_{p}^{2}\left(1-2 M / r_{p}\right)}, \\
C & =\left[\stackrel{(1)}{\Gamma}^{t}{ }_{r r} \dot{r}_{p}^{3}+\left(2{\stackrel{(1)}{\Gamma^{t}}}_{t r}-{\stackrel{(1)}{\Gamma^{r}}}_{r r}\right) \dot{r}_{p}^{2}+\left(\stackrel{(1)}{\Gamma}_{t t}^{t}-2{\stackrel{(1)}{\Gamma^{r}}}_{t r}\right) \dot{r}_{p}-\stackrel{(1)}{\Gamma}_{t t}^{r}\right] \\
& =\sum_{l=0}^{\infty} C_{\ell} .
\end{aligned}
$$

It is precisely $C_{\ell}$ the piece that, being finite for each multipole, diverges when summed over $\ell$ (here we consider the averaged value of $C_{\ell}$ over both radial sides of $r_{p}$ ). The direct numerical integration of the wave equation (3) shows that we can split $C_{\ell}$ as

$$
C=\sum_{\ell=0}^{\infty}\left\{\left(2 \ell+\frac{3}{2}-\beta\right)^{-\beta+1 / 2} C_{\infty}+C_{\ell}^{r e n}\right\},
$$

where the introduction of $\beta$ is motivated by the $D$-dimensional extension of the initial-value problem for time-symmetric conformally flat data. In this case $-\beta=D-\frac{9}{2}$. We neatly determined numerically that $\beta=\frac{1}{2}$ along the trajectory of the particle.

This form of the coefficient $C$, determining the corrected trajectory of the particle, shows clearly that the $\ell$-independent term, summed over $\ell$, diverges. We also determined numerically that $C_{\ell}^{r e n}$ behaves like $\ell^{-2}$ for large $\ell$, thus giving raise to a finite contribution to $C$. The key observation here is that we can bring the divergent sum to the form of the Riemann $\zeta$-function [11], $\zeta(a, b)=\sum_{\ell=0}^{\infty}(\ell+b)^{-a}$,

$$
C=2^{-\beta+1 / 2} C_{\infty} \zeta\left(\beta-\frac{1}{2}, \frac{1}{2}\right)+\sum_{\ell=0}^{\infty} C_{\ell}^{r e n} .
$$

Since the analytically continued $\zeta$-function gives $\zeta\left(0, \frac{1}{2}\right)=0$, in order to regularize $C$, we must just subtract from each multipole their $\ell \rightarrow \infty$ piece. When we do that [10] we have found that the effect of $C^{r e n}$ on the trajectory is to generate a repulsive contribution that has a maximum near the peak of the Zerilli potential at $r=3.1 \mathrm{M}$.

Let us label functions with the \pm superindex to refer to their values in the region $r>r_{p}$ and $r<r_{p}$ near the location of the particle $r_{p}$, respectively. One can see that both, initial data and the Zerilli equation imply the following formal symmetry:

$$
\sqrt{L} \psi_{\ell}^{ \pm}(-L)=\sqrt{L} \psi_{\ell}^{\mp}(L)
$$

where $L=\ell+\frac{1}{2}$. The same symmetry holds for the $t$ and $r$ derivatives, hence the reaction 'force' will have the form $\mathcal{F}^{ \pm}(L)=p\left(L^{2}\right) \pm L q\left(L^{2}\right)$ and the large- $L$ expansion of its average

$$
\begin{aligned}
\left\langle\mathcal{F}_{p}\right\rangle & =\sum_{\ell=0}^{\infty}\left\langle\mathcal{F}_{\ell}\right\rangle=\sum_{\ell=0}^{\infty} p\left(L^{2}\right)=\sum_{\ell=0}^{\infty} \sum_{n=0}^{\infty} \frac{A_{n}}{L^{2 n}}\left\langle\mathcal{F}_{p}\right\rangle \\
& =\sum_{n=0}^{\infty} A_{n} \sum_{\ell=0}^{\infty} L^{-2 n}=\sum_{n=0}^{\infty} A_{n} \zeta\left(2 n, \frac{1}{2}\right) \\
& =A_{0} \zeta\left(0, \frac{1}{2}\right)+A_{2} \zeta\left(2, \frac{1}{2}\right)+A_{4} \zeta\left(4, \frac{1}{2}\right)+\cdots
\end{aligned}
$$


where we observe that $\zeta\left(0, \frac{1}{2}\right)=0$ gives the regularization, $\zeta\left(2, \frac{1}{2}\right)=\frac{1}{2} \pi^{2}$ gives the leading reaction 'force' and $\zeta\left(4, \frac{1}{2}\right)=\frac{1}{6} \pi^{4}$ gives an estimate of the 'error' of the leading term of the order of 7-8\%, assuming analytical knowledge of the $l=0,1$ multipoles.

Barack and co-workers $[13,14]$ have developed independently a similar approach (in the sense that they also decompose into multipoles), but that uses the regularization method of [5] and implement it for the scalar radiation in the Schwarzschild background. Their results are completely compatible with those of the $\zeta$-function regularization in the sense that in the end it is exactly only $\ell \rightarrow \infty$ that has to be subtracted from the originally divergent force.

Barack and Lousto [12] have been able to confirm analytically the numerical behaviour discussed above. Based on a local analysis of the two-point Green function we make a $1 / L$ (with $L=\ell+\frac{1}{2}$ ) expansion to determine the behaviour of the waveform $\psi_{\ell}$ and its derivatives for large $\ell$, for instance

$\psi_{\ell}^{ \pm}\left(r_{p}\right)=4 m_{0} \sqrt{2 \pi} \sqrt{L}\left\{L^{-3} \pm 2 E L^{-4}+\mathcal{O}\left(L^{-5}\right)\right\}$

$\partial_{r} \psi_{\ell}^{ \pm}\left(r_{p}\right)=\frac{4 m_{0} \sqrt{2 \pi}}{r_{p}-2 M} \sqrt{L}\left\{\mp E L^{-2}-\frac{3}{2} E^{2} L^{-3} \pm\left(\frac{6 M}{r_{p}}-\frac{9}{4}\right) E L^{-4}+\mathcal{O}\left(L^{-5}\right)\right\}$

$\partial_{t} \psi_{\ell}^{ \pm}\left(r_{p}\right)=-\left(1-2 M / r_{p}\right)\left(\frac{\dot{r}_{p}}{E}\right) \partial_{r} \psi_{\ell}^{ \pm}\left(r_{p}\right)$

for a particle released from rest at $r_{0}, E=\sqrt{1-2 M / r_{0}} . \pm$ denotes the side derivatives concerning $r_{p}$ (waveforms are discontinuous). Hence we can compute

$$
C_{\ell}^{ \pm}=a^{ \pm} L+b+c^{ \pm} L^{-1}+\mathrm{O}\left(L^{-2}\right),
$$

where $a^{ \pm}, b$ and $c^{ \pm}$are $l$-independent coefficients. We find that the average value of $a$ and $c$ vanish at the location of the particle, while the average

$$
\langle C(l \rightarrow \infty)\rangle=-\frac{\sqrt{\pi} m_{0}}{r^{2}}\left(1-2 M / r_{p}\right)^{3 / 2} E^{-2} .
$$

This method can be used to assist the numerical computation for large $\ell$ carrying out the expansion to order $1 / L^{2}$ in $C$. Thus, making the numerical integration necessary for only the first few lower multipoles. This might be crucial when dealing with generic orbits around Schwarzschild black holes. The analytic expansions would allow a clean application of the $\zeta$-regularization scheme and sum over large $\ell$ of the regularized reaction 'force'.

In the orbital case it will also be important to test our final results with those of the energy-balance approximation [15-17]. This method provides accurate results for circular orbits on the Kerr background [16]. Our method, in principle, could also be implemented to a particle orbiting a Kerr hole since the Teukolsky equation that describes the perturbations around rotating holes, can be decomposed into multipoles in the frequency domain (Laplace decomposition of the time dependence [18]). The details of this implementation remain to be explicitly worked out.

A further application of the above results one can foresee is the extension of the analysis to second perturbative order [19]. In this way one can increase the accuracy with which waveforms are computed as well as reaching not so small mass ratios $m / M$. At this stage, the resulting waveforms will not only be relevant for LISA, but also for ground-based gravitational wave detectors sensitive to galactic binary black holes of comparable masses and black hole-neutron star systems.

I would like to end this contribution with an optimistic note from the theoretical side and predict that the subsequent progress in our understanding of radiation reaction will soon bring good news for modelling gravitational emission from astrophysical sources. 


\section{Acknowledgment}

I am grateful to L Barack for many useful remarks.

\section{Appendix. Connection coefficients}

Here we make explicit the details that enter into the computation of $C_{\ell}$ given in equation (7). The connection coefficients involved in the first-order geodesic, equation (4) in terms of the first-order perturbations as defined in the Regge-Wheeler (RW) gauge [3] are

$$
\begin{aligned}
\Gamma_{t t}^{t} & =\frac{M}{r^{2}} H_{1}-\frac{1}{2} \dot{H}_{0}, \quad \Gamma_{t r}^{t}=\frac{M}{r^{2}}\left(1-\frac{2 M}{r}\right)^{-1}-\frac{1}{2} H_{0}^{\prime}, \\
\Gamma_{r r}^{t} & =\left(1-\frac{2 M}{r}\right)^{-2}\left[\frac{1}{2} \dot{H}_{2}-\left(1-\frac{2 M}{r}\right) H_{1}^{\prime}-\frac{M}{r^{2}} H_{1}\right], \\
\Gamma_{t t}^{r} & =\left(1-\frac{2 M}{r}\right)\left[\dot{H}_{1}+\frac{M}{r^{2}}\left(1-H_{2}-H_{0}\right)-\frac{1}{2}\left(1-\frac{2 M}{r}\right) H_{0}^{\prime}\right], \\
\Gamma_{t r}^{r} & =-\frac{M}{r^{2}} H_{1}+\frac{1}{2} \dot{H}_{2}, \quad \Gamma_{r r}^{r}=-\frac{M}{r^{2}}\left(1-\frac{2 M}{r}\right)^{-1}+\frac{1}{2} H_{2}^{\prime},
\end{aligned}
$$

where the overdot and prime denote the derivative with respect to the Schwarzschild time $t$ and radial coordinate $r$, respectively. In addition, we have from Einstein's equations in the RW gauge (see [4], equation $(\mathrm{C} 7 \mathrm{~g})$ ), that for a radial infall

$$
H_{0}=H_{2}, \quad \ell \geqslant 2 \text {. }
$$

This allows us to write everything in terms of $H_{1}^{\ell}$ and $H_{2}^{\ell}$ only (and its derivatives). $K^{\ell}$ does not appears in the head-on geodesics.

Having proven the continuity of the metric coefficients at $r=r_{p}$ our way is clear to compute the trajectory of the particle to first perturbative order. Our last step is to compute these metric derivatives in terms of $\psi$ and $\dot{\psi}$. Since we know that the metric coefficients are continuous, their derivatives will have a jump, but not delta (or derivatives of it) terms. Then, in order to simplify expressions we will not write the source terms and use an overbar $(\bar{\psi})$ on radial derivatives of $\psi$ to indicate that we have already subtracted its singular behaviour at $r=r_{p}$ and taken the average value.

The $r$-derivative of $H_{2}$ from [10] is

$$
\begin{aligned}
\partial_{r} H_{2}=(r-2 M) \partial_{r}^{3} \bar{\psi}+\left[1+\frac{\lambda r^{2}-\lambda M r+3 M^{2}}{(\lambda r+3 M) r}\right] \partial_{r}^{2} \bar{\psi} \\
+\frac{-\lambda^{2}(\lambda+1) r^{3}-\lambda(2 \lambda-3) M r^{2}-15 \lambda M^{2} r-18 M^{3}}{r^{2}(\lambda r+3 M)^{2}} \partial_{r} \bar{\psi} \\
+\frac{\lambda^{3}(\lambda+1) r^{4}+3 \lambda^{2}(\lambda-1) M r^{3}+27 \lambda^{2} M^{2} r^{2}+63 \lambda M^{3} r+54 M^{4}}{r^{3}(\lambda r+3 M)^{3}} \bar{\psi} .
\end{aligned}
$$

Likewise, upon $r$-derivation of the expression for $H_{1}$, given in [10] we obtain

$$
\begin{aligned}
\partial_{r} H_{1}=r \partial_{r}^{2} \dot{\bar{\psi}} & +\frac{2 \lambda r^{2}+(3-5 \lambda) M r-9 M^{2}}{(r-2 M)(\lambda r+3 M)} \partial_{r} \dot{\bar{\psi}} \\
& +\frac{\left[\lambda(\lambda+3) r^{2}-6 \lambda M r+3(4 \lambda+3) M^{2}\right] M}{(r-2 M)^{2}(\lambda r+3 M)^{2}} \dot{\bar{\psi}} .
\end{aligned}
$$


The time derivative of $\mathrm{H}_{2}$ can also be obtained

$$
\begin{aligned}
\partial_{t} H_{2}=(r-2 M) \partial_{r}^{2} \dot{\bar{\psi}}+\frac{3 M^{2}-\lambda M r+\lambda r^{2}}{r(\lambda r+3 M)} \partial_{r} \dot{\bar{\psi}} \\
-\frac{9 M^{3}+9 \lambda M^{2} r+3 \lambda^{2} M r^{2}+\lambda^{2}(\lambda+1) r^{3}}{r^{2}(\lambda r+3 M)^{2}} \dot{\bar{\psi}}
\end{aligned}
$$

and finally we find $\partial_{t} H_{1}$ from Zerilli's [4] equation (C7e), where $K^{\prime}$ can be found from $H_{2}$, given in [10] and $H_{2}^{\prime}$ is given by equation (A3),

$$
\begin{aligned}
\partial_{t} H_{1}=\frac{(r-2}{r} & M)^{2} \\
& -2\left(2 \lambda^{2} M r^{3}+18 \lambda M^{2} r^{2}+27 r M^{3}-9 \lambda^{2} M^{2} r^{2}-42 \lambda M^{3} r\right. \\
& \left.-63 M^{4}+\lambda^{3} r^{4}+\lambda^{2} r^{4}-2 M \lambda^{3} r^{3}\right) /\left(r^{3}(\lambda r+3 M)^{2}\right) \partial_{r} \bar{\psi} \\
& +2\left(-189 M^{5}+81 M^{4} r-216 \lambda M^{4} r+90 \lambda M^{3} r^{2}-90 \lambda^{2} M^{3} r^{2}\right. \\
& \left.+33 \lambda^{2} M^{2} r^{3}-18 \lambda^{3} M^{2} r^{3}-3 \lambda^{3}(\lambda-1) M r^{4}+\lambda^{3} r^{5}+\lambda^{4} r^{5}\right) \bar{\psi} \\
& \times\left(r^{4}(3 M+\lambda r)^{3}\right)^{-1} .
\end{aligned}
$$

This expression completes the equations needed to (numerically) integrate the geodesic equation (4) that gives us the particle's trajectory to first perturbative order. Note that the numerical implementation should be able to handle third-order derivatives of $\psi_{\ell}$.

\section{References}

[1] Ghez A, Morris M, Becklin E E, Kremenek T and Tanner A 2000 Nature 407349 Preprint astro-ph/0009339

[2] Danzmann K et al 1998 LISA: proposal for a laser-interferometric gravitational wave detector in space MaxPlanck-Institut für Quantum Optics Report MPQ 233

[3] Regge T and Wheeler J A 1957 Phys. Rev. 1081063

[4] Zerilli F J 1970 Phys. Rev. Lett. 24737

[5] Mino Y, Sasaki M and Tanaka T 1997 Phys. Rev. D 553457

[6] Quinn T C and Wald R 1997 Phys. Rev. D 563381

[7] Lousto C O and Price R 1997 Phys. Rev. D 552124

[8] Lousto C O and Price R 1997 Phys. Rev. D 566439

[9] Lousto C O and Price R 1998 Phys. Rev. D 571073

[10] Lousto C O 2000 Phys. Rev. Lett. 845251

[11] Birrell N D and Davies P C W 1982 Quantum Fields in Curved Space (Cambridge: Cambridge University Press)

[12] Barack L and Lousto C O 2001 in preparation

[13] Barack L and Ori A 2000 Phys. Rev. D 61 061502(R) Barack L 2000 Phys. Rev. D 62084027

[14] Barack L and Burko L M 2000 Phys. Rev. D 62084040 Burko L M 2000 Phys. Rev. Lett. 844529

[15] Cutler C, Kennefick D and Poisson E 1994 Phys. Rev. D 503816

[16] Hughes S A 2000 Phys. Rev. D 62044029

[17] Finn L S and Thorne K S 2000 Phys. Rev. D 62124021

[18] Campanelli M and Lousto C O 1998 Phys. Rev. D 58024015

[19] Campanelli M and Lousto C O 1999 Phys. Rev. D 59124022 